\title{
Reporting plant hormone levels: a disappearing act
}

\section{A fluorescent protein fusion reports levels of the plant hormone auxin by succumbing to its degradation signal.}

Charles Darwin noticed that the canary grass he used to feed his birds bent eagerly toward light from the window. He and his son Francis carried out experiments describing a mysterious 'influence' that originated in the young shoot tips and was responsible for bending in the region below. We now know that the culprit is a class of plant hormones called auxins. Visualizing these small moving targets has posed a difficult challenge, but Teva Vernoux at the École Normale Supérieure in Lyon and his colleagues now have designed a reporter to quantify auxin levels at high resolution in both space and time in the thale cress, Arabidopsis thaliana.

Auxins are multifaceted molecules that signal from within the cell to control cell expansion, polarization and differentiation. They can trigger organ outgrowth and guide tissue patterning in the embryo, shoot, flower and root in addition to driving movements such as the shoot bending described by the Darwins or root bending in response to gravity.

To command all of these roles, auxins are shuttled from cell to cell with fine spatial precision, and signaling has evolved to be very rapid. Auxin controls target gene expression by prompting the degradation of inhibitors that bind to transcription factors and repress their activity. Most of what we know about auxin localization comes from using a binding site for these transcription factors-DR5-to control the expression of GFP. Although this approach has been enormously useful, it only captures signaling output. Where is auxin itself?

Reasoning that auxin physically interacts with the degradation motif of the inhibitors (DII), Vernoux and colleagues fused such a motif to Venus fluorescent protein to give a direct and quantitative readout of auxin levels. High levels of the reporter are maintained in cells by a ubiquitous promoter, and elevated auxin levels cause a drop in fluorescence.

One advantage of the strategy is speed. "Degradation is something that occurs extremely rapidly," says Vernoux. "That means that when you have an increase in auxin, you can follow it almost in real time." The auxin signal leads to transcription within minutes, which is captured by DII-Venus fluorescence kinetics, whereas fluorescence from the DR5 reporter lags by 2 hours because of the time needed to make GFP. However, to report a drop in auxin, DII-Venus also experiences a lag for translation and folding (though it helps that Venus is a rapidly folding protein).

Images of DII-Venus expression are like a photographic negative of auxin levels, and reporting a loss of signal depends on homogenous baseline expression. The authors examined baseline expression using a control reporter based on a mutation in DII that makes it insensitive to auxin. It showed limited variability across root and shoot tissues and in different plants, and the researchers used the levels of the control reporter to normalize DII-Venus signal.

Vernoux sees their tool as complementary to existing reporters. "One monitors the input; the other gives you some idea of transcriptional response to auxin," he notes. "So even without having any information on the pathway in-between, you know how the cell processes the signal." Imaging of DII-Venus revealed higher auxin levels in a zone of the root where cells stop dividing, and changing the root's orientation to gravity caused a rapid loss of DII-Venus in several tissues on the lower side of the root. These observations differ from studies using the DR5 reporter; interpreting this difference should yield

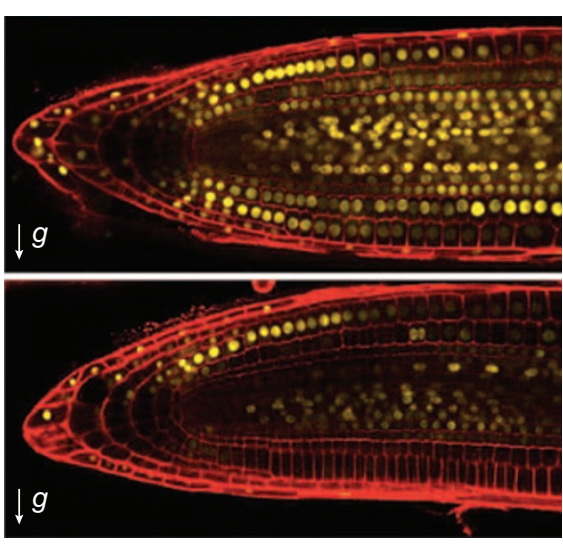

A rapid auxin sensor. Turning an Arabidopsis root on its side (top) causes a loss of DII-Venus signal in response to elevated auxin levels (bottom, 30 min later). $g$, direction of gravity. Reprinted from Nature.

interesting insights into how the auxin signal is processed.

The group also imaged DII-Venus in the shoot tip and found high auxin levels in the stem cells that they did not detect with the DR5 reporter. Although Venus is not highly photostable, DII-Venus could be imaged every 2-5 minutes for hours without substantial loss of fluorescence.

The reporter strategy can be used to follow degradation in other systems, and the group is working on sensors for other plant hormone signaling pathways that also rely on degradation. Vernoux is excited by the ability to directly visualize dynamic changes in auxin levels. "This comes down to being able to understand exactly what changes in the concentration of hormones like auxin trigger in terms of cell behavior," he says.

\section{Tal Nawy}

\section{RESEARCH PAPERS}

Brunoud, G. et al. A novel sensor to map auxin response and distribution at high spatio-temporal resolution. Nature 482, 103-106 (2012). 